\title{
The E3 ligase MuRF2 plays a key role in the functional capacity of skeletal muscle fibroblasts
}

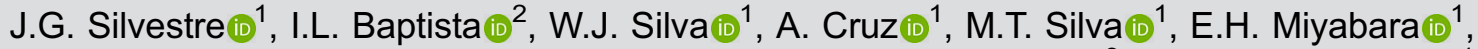 \\ S. Labeit $\left(^{3}\right)^{3}$, and A.S. Moriscot ${ }^{1}{ }^{1}$ \\ ${ }^{1}$ Departamento de Anatomia, Instituto de Ciências Biomédicas, Universidade de São Paulo, São Paulo, SP, Brasil \\ ${ }^{2}$ Faculdade de Ciências Aplicadas, UNICAMP, Limeira, SP, Brasil \\ ${ }^{3}$ Institute for Integrative Pathophysiology, Mannheim Medical University, Faculty for Clinical Medicine Mannheim, \\ University of Heidelberg, Mannheim, Germany
}

\begin{abstract}
Fibroblasts are a highly heterogeneous population of cells, being found in a large number of different tissues. These cells produce the extracellular matrix, which is essential to preserve structural integrity of connective tissues. Fibroblasts are frequently engaged in migration and remodeling, exerting traction forces in the extracellular matrix, which is crucial for matrix deposition and wound healing. In addition, previous studies performed on primary myoblasts suggest that the E3 ligase MuRF2 might function as a cytoskeleton adaptor. Here, we hypothesized that MuRF2 also plays a functional role in skeletal muscle fibroblasts. We found that skeletal muscle fibroblasts express MuRF2 and its siRNA knock-down promoted decreased fibroblast migration, cell border accumulation of polymerized actin, and down-regulation of the phospho-Akt expression. Our results indicated that MuRF2 was necessary to maintain the actin cytoskeleton functionality in skeletal muscle fibroblasts via Akt activity and exerted an important role in extracellular matrix remodeling in the skeletal muscle tissue.
\end{abstract}

Key words: Fibroblast; Skeletal muscle; Migration; MuRF2; E3 ligase; Wound healing

\section{Introduction}

Mesenchymal cells are known for giving rise to a large number of connective tissue cells such as myoblasts, chondroblasts, adipocytes, osteoblasts, and fibroblasts. Fibroblasts, in turn, are a highly heterogeneous population of cells, and can be found in different tissues (1), being the most abundant stromal cell type (2). The extracellular matrix $(E C M)$, produced by fibroblasts, plays essential roles in preserving the structural integrity of connective tissues and during healing progression (1). In skeletal muscle tissue, fibroblasts are one of the most abundant cell population (3). The ECM is an important scaffold for skeletal muscle fibers and is involved in force transmission and protection against mechanical trauma (4).

Regeneration of skeletal muscle is a highly complex process that involves the activation of a large number of intracellular and cell-to-cell responses $(5,6)$. Although it is widely known that the regeneration process relies upon satellite cells in order to repair the skeletal muscle fiber and recover function, the much less understood fibroblasts also play an important role. Recently, there has been increasing evidence of a role for the ECM in signaling through regulatory molecules (7). Interestingly, however, it is known that excessive ECM can impair muscle function and hinder muscle regeneration $(8,9)$. In wound repair, fibroblasts proliferate and differentiate into active states, secreting important components of the ECM. These cells also intensely engage in migration and remodeling and exert traction forces in the ECM (10).

The migratory capacity of fibroblasts is crucial for matrix deposition and wound healing (11), and recent studies highlight the importance of correct cytoskeleton arrangement for fibroblast migration. Noteworthily, it has been shown that Akt activity is crucial for the establishment of actin filaments at the edge of fibroblasts during the migration process (12). Recently, it has been shown that E3 ubiquitin ligases play important roles in cytoskeleton dynamics towards proper migration (13).

The E3 ubiquitin ligases are proteins that play essential roles in cellular homeostasis and in the degradation of mutated misfolded or damaged proteins $(14,15)$. In skeletal muscle tissue, an important family of E3 ubiquitin ligases initially named muscle-specific RING finger (MuRFs) has been described (16). Subsequently, MuRFs were categorized as MuRF1 (17) and MuRF2 (18). MuRF2 can establish 
direct binding with stable microtubules and proteins at the M-line region, and the silencing of MuRF1 and MuRF2 disrupts the structure of microtubules and intermediate filaments (18).

Thus, due to the common progenitor shared by fibroblasts and skeletal muscle cells, the large number of fibroblasts present in skeletal muscle niche, the importance of cytoskeleton organization for fibroblasts migration, and the role of MuRF2 in the stabilization of microtubules, our study aimed to test whether MuRF2 is expressed and if it plays a functional role in skeletal muscle fibroblasts.

\section{Material and Methods}

The protocols employed in this study followed the ethical guidelines in animal research (Brazilian College of Animal Experimentation). These protocols were submitted and approved by the Institute of Biomedical Sciences/ University of São Paulo - Ethical Committee for Animal Research (\# 118/13).

\section{Fibroblasts and myogenic cell culture}

Purified cells were obtained from primary culture using a modified version of a previously described preplating technique (19). Cells were isolated from the hind limb muscles of male FVB mice ( $\sim 8$ weeks old, $30 \pm 3 \mathrm{~g}$ ). Cell isolation and digestion were made as previously described $(20,21)$. After digestion, the cells were incubated at $37^{\circ} \mathrm{C}$ in an atmosphere of $5 \% \mathrm{CO}_{2}$ for $40-50 \mathrm{~min}$ on $75 \mathrm{~cm}^{2}$ tissue culture dishes. The supernatant was used as myoblastenriched primary skeletal muscle culture (see below) and the rapidly adhering fibroblasts were maintained (P0) as previously reported (19). These fibroblast populations were kept up to $80-90 \%$ confluence and then passed using the same protocol described above, always discarding the nonadherent supernatant cells. This procedure was repeated 3 times. All experiments using fibroblasts were performed at the third passage (P3).

Both C2C12 (ATCC CRL-1772, USA) and the supernatant cells from P0 myoblast-enriched pool were plated in Matrigel-coated 24-well plates (Matrigel Matrix Growth Factor Reduced; BD Biosciences \#354230, USA) and incubated until 2 days after differentiation as previously described $(20,21)$.

\section{Cardiac fibroblasts}

Primary cardiac fibroblasts were obtained as previously described (22) and kindly provided by Dr. Maria Luiza Morais Barreto-Chaves (Department of Anatomy, University of São Paulo, Brazil).

\section{Mouse embryonic fibroblasts}

Mouse embryonic fibroblasts (MEFs, ATCC\#SCRC1008) were kindly provided by Prof. Dr. Julio Cesar Batista Ferreira (Department of Anatomy, University of
São Paulo, Brazil). These cells were maintained in DMEM supplemented with $10 \%$ FBS until $\sim 90 \%$ confluency, and subsequently MuRF2 immunofluorescence was carried out.

\section{Pancreatic islets}

Pancreatic islets were kindly provided by Angelo R. Carpinelli (Department of Physiology and Biophysics, University of São Paulo, Brazil). Islets were obtained from Wistar rats as previously described (23).

\section{siRNA silencing}

For small interfering RNA (siRNA) interference assays, we transfected fibroblasts at $80-90 \%$ confluence with scrambled (\#AM4613, Ambion, USA) and MuRF2 (cat \#AM4390771, Ambion) siRNAs. Fibroblasts plated on 24well plates were transfected with $30 \mathrm{nM}$ siRNAs using siPORT $^{\mathrm{TM}}$ NeoFX ${ }^{\mathrm{TM}}$ transfection agent (Ambion \#AM4511) according to the manufacturer's instructions and as previously described (20). The cells were transfected for $12 \mathrm{~h}$, and then the siRNA solution was replaced with DMEM containing $10 \%$ FBS.

\section{Wound healing assay}

Wound healing assays were performed based on a previously described method (24). In vitro scratch wounds were created $12 \mathrm{~h}$ after siRNA silencing by applying a straight cut on confluent cell monolayers with a sterile disposable pipette tip. Four independent experiments were performed.

\section{Western blot analysis}

Cells were collected and protein lysates were prepared for western blot analysis as previously described (20).

Primary antibodies used were: MuRF2 (18); Akt (Cell Signaling \#9272, USA); GAPDH (Cell Signaling \#2118); pAkt Ser473 (Cell Signaling \#4058); pAkt Thr308 (Cell Signaling \#4056). Secondary antibodies used were: Goat anti-rabbit (Jackson ImmunoResearch \#111-035-003, USA). After the membranes were washed, specific bands were visualized with the Fusion Fx (Vilber Lourmat Biosystems, France) system after the use of the sensitive chemiluminescent substrate Lumina ${ }^{\mathrm{TM}}$ Forte Western HRP Substrate (Millipore, USA). Loading variations were monitored by Ponceau staining and GAPDH. The relative protein expression intensities were quantified by densitometry using ImageJ (1.48 version, Wayne Rasband, National Institutes of Health, USA).

\section{Immunofluorescence}

Cells on a 24 -well plate were fixed with $4 \%$ paraformaldehyde for $10 \mathrm{~min}$ at room temperature, washed with PBS containing $0.1 \%$ Triton $\mathrm{X}-100$ (PBS-T) two times for $3 \mathrm{~min}$ each and then blocked/permeabilized with PBS-T containing 1\% BSA for $1 \mathrm{~h}$. Then, the wells were prepared for immunofluorescence analysis as previously 
described (20). Fluorescence micrographs were obtained with the microscope Zeiss Imager M1 (Carl Zeiss Microscopy $\mathrm{GmbH}$, Germany) equipped with FITC, rhodamine, and FURA filters. Primary antibodies used were: MuRF2 (18), MyoD (sc-31940), Ki-67 (NB110-89717), and TCF-4 (sc-13027). Secondary antibodies used were: Cy3 Donkey Anti-rabbit (Jackson ImmunoResearch \#711165-152), Cy3 Donkey Anti-goat (Jackson ImmunoResearch \#705-175-147), and Cy2 Goat Anti-rabbit (Jackson ImmunoResearch \#111-225-008). Digital images from a Zeiss Axiocam MRn were exported as TIFF files with Axiovision 4.6 software without any subsequent processing in parameters such as contrast and brightness.

\section{Proliferation index}

This assay was performed following the same protocol used in the wound healing assay. The proliferation index is reported as the percentage of Ki67-labeled cells (red)/ DAPI-labeled cells (blue) over the total number of cells in five fields/well (two wells per group).

\section{Detection of polymerized actin-containing lamellipodia}

This assay was performed $24 \mathrm{~h}$ after the onset of wound healing assays and following the same specification used in immunofluorescence analysis using phalloidinAlexa Fluor 488 (A12379, Invitrogen, USA). The number of positive cells is reported as the percentage of polymerized actin-containing lamellipodia (densely green at the edge of the cell)/DAPI-labeled cells (blue) over the total number of cells in six fields/well (two wells per group).

\section{Quantitative polymerase chain reaction and semi- quantitative PCR}

Cells and tissues were homogenized, and total RNA was obtained using TRIzol reagent (Invitrogen) following the manufacturer's instructions. One microgram of total RNA was used and cDNA was synthesized by cDNA Promega kit (M-MLV-Promega, USA). For semi-quantitative PCR, we used 50 ng of cDNA, amplified by 40 cycles of PCR using Taq DNA polymerase (Sinapse Inc, Brazil).

Real-Time PCR was performed separately for each gene in duplicates in a $25-\mu \mathrm{L}$ reaction volume (20 ng cDNA, $12.5 \mu \mathrm{L}$ Sybr Green Master Mix ${ }^{\circ}$, Applied Biosystems, USA) and $300 \mathrm{nM}$ of each primer. Primer sequences were as follows: MuRF1 forward, CCTGCTGGTGGAAAACATCA, and MuRF1 reverse GGCTATTCTCCTTGGTCACTCT; MuRF2 forward, GTCCTGGTGACACAGATTGGAT, and MuRF2 reverse, TGCTGCCTATGTGCTTCTCA; collagen 1 forward, CTTGGTGGTTTTGTATTCGATGAC, collagen 1 reverse, GCGAAGGCAACAGTCGCT; cyclophilin forward, GCCGATGACGAGCCCTTG, and cyclophilin reverse, TGCCGCCAGTGCCATTAT. Cyclophilin expression served as an internal control and relative levels of $\mathrm{mRNA}$ expression were normalized to its expression.

\section{Statistical analysis}

The data are reported as means $\pm S E$ from three independent experiments. Statistical analysis was performed by Student's unpaired $t$-test between groups using GraphPad Prism v.7 (GraphPad Software, USA). For all comparisons, $\mathrm{P}<0.05$ was considered significant.

\section{Results}

\section{Validation of fibroblast culture}

Our first aim was to confirm the degree of fibroblast pureness in our cultures. Accordingly, we observed no expression of the myoblast myogenic marker MyoD (Figure $1 \mathrm{~A}$ and $\mathrm{B}$ ). Furthermore, as a positive control, we detected MyoD expression in the cells from $\mathrm{PO}$ myoblastenriched pool of primary myoblasts (Figure $1 \mathrm{C}$ and $\mathrm{D}$ ). Finally, we observed no formation of myotubes in our skeletal muscle fibroblasts (Figure $1 \mathrm{E}$ and F), nonetheless, as expected, myotube formation was confirmed when using differentiation medium in the cells from $\mathrm{PO}$ myoblast-enriched primary myoblasts pool (Figure 1G and $\mathrm{H}$ ). In order to confirm the fibroblast purity, we aimed to detect TCF4 (transcription factor 7-like 2, Tcf7L2Genome Mouse Browser), which is considered a hallmark of the fibroblast phenotype $(5,25)$. The analysis showed that $100 \%$ of skeletal muscle fibroblasts were positive to TCF4 (Figure $1 \mathrm{~K}, \mathrm{~L}$ and $\mathrm{M}$ ) while, as expected, C2C12 showed no labeling to TCF4 (Figure $1 \mathrm{I}$ and J) confirming that skeletal muscle fibroblasts used in this study do not contain myogenic cells.

\section{MuRF2 was expressed in fibroblasts}

To verify expression of MuRF1 and MuRF2 in skeletal muscle fibroblasts, we performed a semi-quantitative PCR using $\mathrm{C} 2 \mathrm{C} 12$ as a positive control, lung and pancreatic islets as negative controls, using primers for MuRF1 and MuRF2, generating amplicons of 323 and $193 \mathrm{bp}$, respectively. We observed that skeletal muscle fibroblasts did not express MuRF1 as well as lung and pancreatic islets (Figure 2A). Cyclophilin was used as a housekeeping gene (Figure 2C). These results were expected as previously shown (17). On the other hand, we found MuRF2 expression in skeletal muscle fibroblasts, along with $\mathrm{C} 2 \mathrm{C} 12$ (a purified skeletal muscle cell line) in mouse lung tissue (Figure 2B). Then, we analyzed the cell localization of MuRF2 in three different fibroblast cells by immunofluorescence. Our results showed that MuRF2 was scattered within the cytoplasm, with perinuclear accumulation in skeletal muscle fibroblasts (Figure 2D and G). In primary cardiac fibroblast cells, similarly, we found MuRF2 labeling scattered within the cytoplasm, with perinuclear accumulation (Figure $2 \mathrm{E}$ and $\mathrm{H}$ ). Moreover, when using a commonly used fibroblast cell line (MEFs), we identified a strong labeling in MEFs nucleus, by immunofluorescence for MuRF2 (Figure 2F and I). Thus, our immunofluorescence analysis showed that MuRF2 was present in fibroblasts of different tissue sources. 

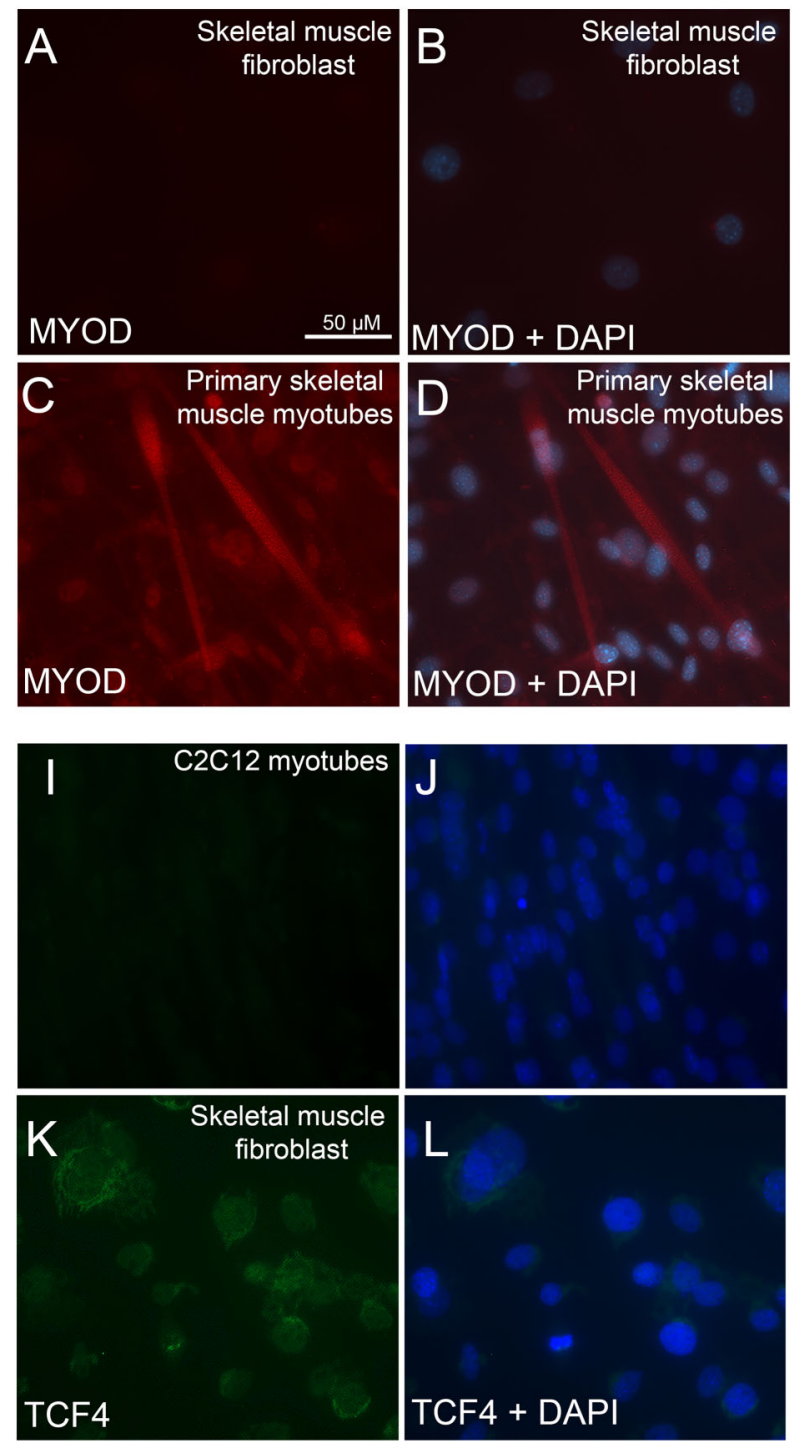
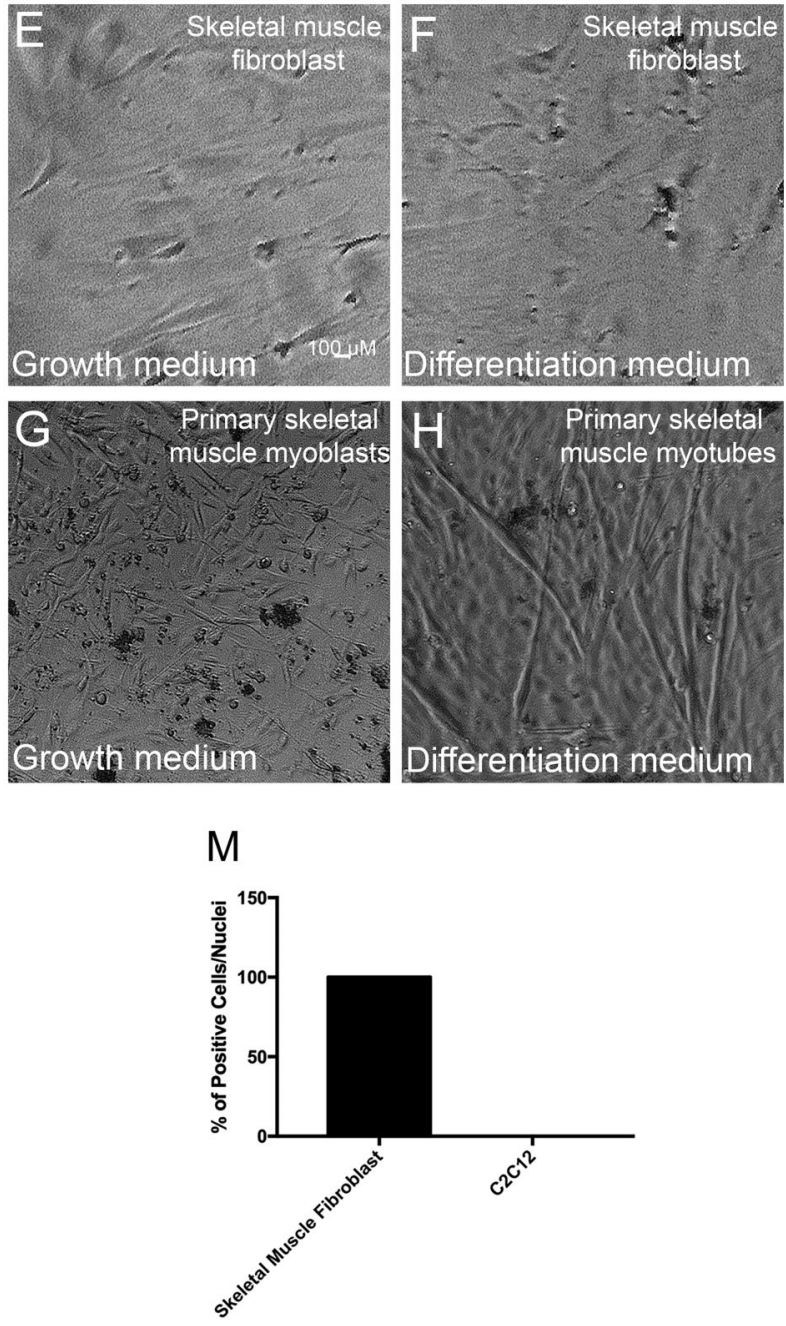

Figure 1. Validation of fibroblast culture. Immunofluorescence for $M y o D$ in skeletal muscle fibroblasts (A, B) and primary skeletal muscle myotubes as a positive control for MyoD labelling (C, D) 2 days after differentiation medium $(2 \% \mathrm{HS})$. Phase contrast microscope image from skeletal muscle fibroblasts submitted to either growth medium with $80-90 \%$ confluence $(\mathbf{E})$ or differentiation medium for 2 days $(\mathbf{F})$. Myoblasts were also submitted to either growth medium with $80-90 \%$ confluence $(\mathbf{G})$ or differentiation medium for 2 days $(\mathbf{H})$ as a positive control. Immunofluorescence for TCF4 in C2C12 myotubes differentiated for 2 days (I and $\mathbf{J})$ and in skeletal muscle fibroblasts (K and $\mathbf{L})$. M: Percentage of TCF4-positive cells. Images were taken at high (A-D and $\mathbf{I} \mathbf{L}, 400 \times$; bar, $50 \mu \mathrm{M})$ and low $(\mathbf{E}-\mathbf{H}, 40 \times$; bar, $100 \mu \mathrm{M})$ magnifications.

In order to confirm the MuRF2 expression in skeletal muscle fibroblasts, we knocked-down MuRF2 using siRNA (MuRF2 siRNA) and found a decrease in both mRNA and protein expression compared to the scrambled (SCRBd) group (Figure $2 \mathrm{~J}, \mathrm{~K}$, and $\mathrm{L}$ ).

\section{Knock-down of MuRF2 impaired skeletal muscle fibroblast wound healing capacity}

To gain insight into the role of the E3 ligase MuRF2 in skeletal muscle fibroblast, we conducted wound healing assays using MuRF2 siRNA. The knockdown of MuRF2 caused a significant decrease in the fibroblast migration capacity after 4 and $24 \mathrm{~h}$ (Figure 3D, H, and I).

Next, we addressed whether the silencing of MuRF2 induced changes in the proliferation of skeletal muscle fibroblasts using Ki67 immunolabeling (Figure 3J-R). Our results showed that MuRF2 siRNA caused a significant increase in Ki67-positive nuclei abundance after $24 \mathrm{~h}$ (Figure $3 \mathrm{Q}$ and $\mathrm{R}$ ). 

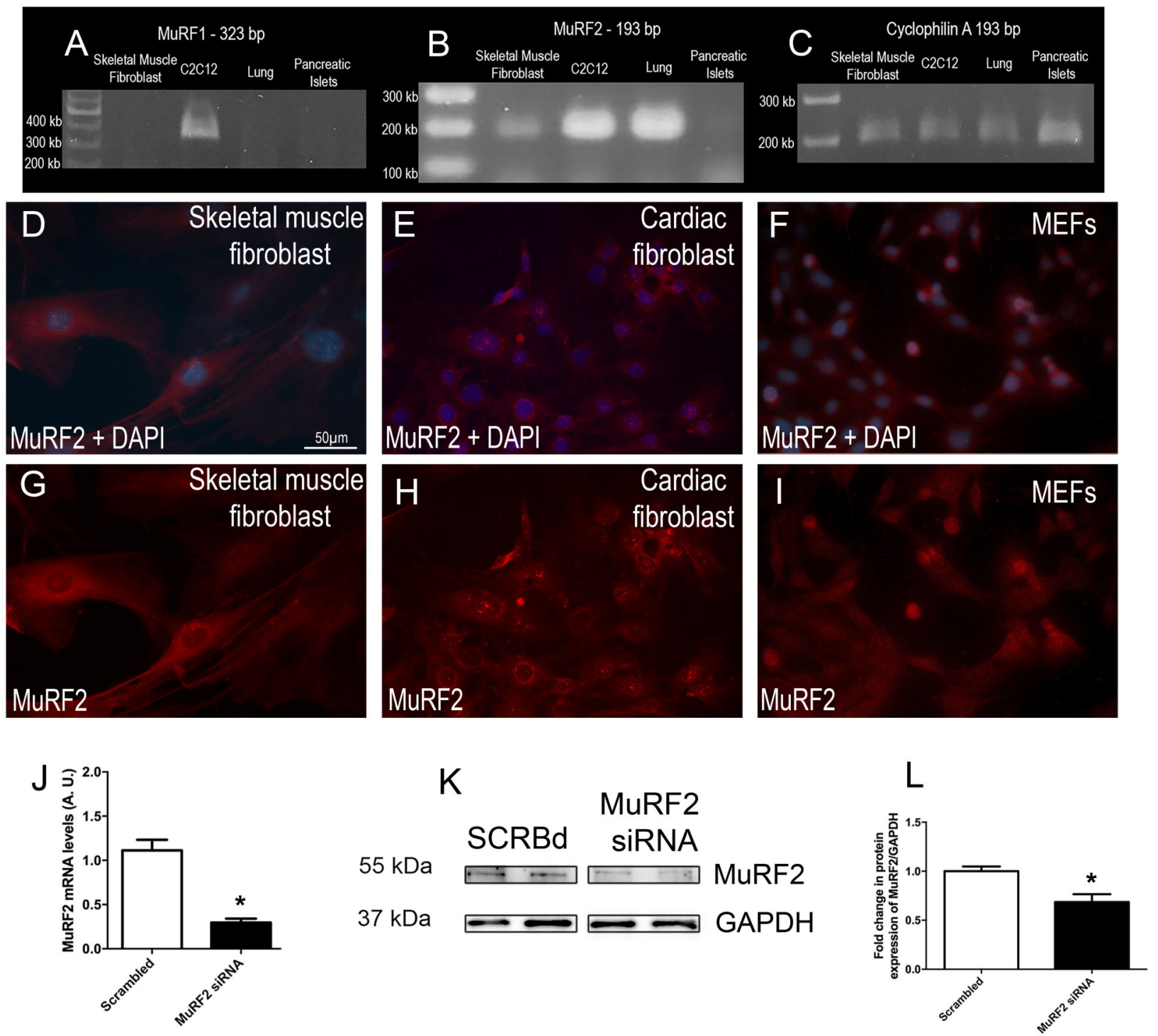

Figure 2. MuRF2 is expressed in fibroblasts. Semi-quantitative PCR for MuRF1 (A) and MuRF2 (B), and cyclophilin as a housekeeping gene (C) in skeletal muscle fibroblasts, C2C12, lung, and pancreatic islets. Immunofluorescence for MuRF2 in skeletal muscle fibroblasts (D and G), cardiac fibroblasts (E and $\mathbf{H}$ ), and mouse embryonic fibroblasts (MEFs) (F and I). J: Real-time PCR in skeletal muscle fibroblasts treated with scrambled (SCRBd) or MuRF2 siRNA. K: Representative immunoblots for MuRF2 and GAPDH in skeletal muscle fibroblasts treated with SCRBd or MuRF2 siRNA. The images belong to the same membrane. L: Relative band intensities were determined by densitometry. Scale bar, $50 \mu \mathrm{M}(\mathrm{D}-\mathrm{I})$. Data are reported as means $\pm \mathrm{SE}$. ${ }^{*} \mathrm{P}<0.05$ vs SCRBd (Student's $t$-test).

\section{Knock-down of MuRF2 in skeletal muscle fibroblast impaired lamellipodia formation}

Given that MURF2 is developmentally associated with microtubules (26), we hypothesized that lack of MuRF2 could lead to cytoskeleton disorganization. Therefore, we performed wound healing assays after $12 \mathrm{~h}$ of MuRF2 knock-down. Twenty-four hours after the onset of wound healing assays, the incidence of lamellipodia was analyzed using fluorescent dye conjugated to phalloidin-Alexa Fluor 488 to detect polymerized actin. Figure $4 \mathrm{~A}, \mathrm{~B}$, and $\mathrm{E}$ shows the normal distribution of actin fibers in the SCRBd group, in which the lamellipodia are clearly visible. When MuRF2 was knocked-down, skeletal muscle fibroblasts exhibited a reduction in lamellipodia formation $(\sim 50 \%$ reduction, $\mathrm{P}<0.05$ ) (Figure $4 \mathrm{C}, \mathrm{D}$, and $\mathrm{E}$ ), compromising the organization of the cytoskeleton that is essential for cell migration (27).

Because the Akt Ser473 and Akt Thr308 residues are important in cell migration $(12,24)$, we decided to address their level of phosphorylation in MuRF2 knocked-down 


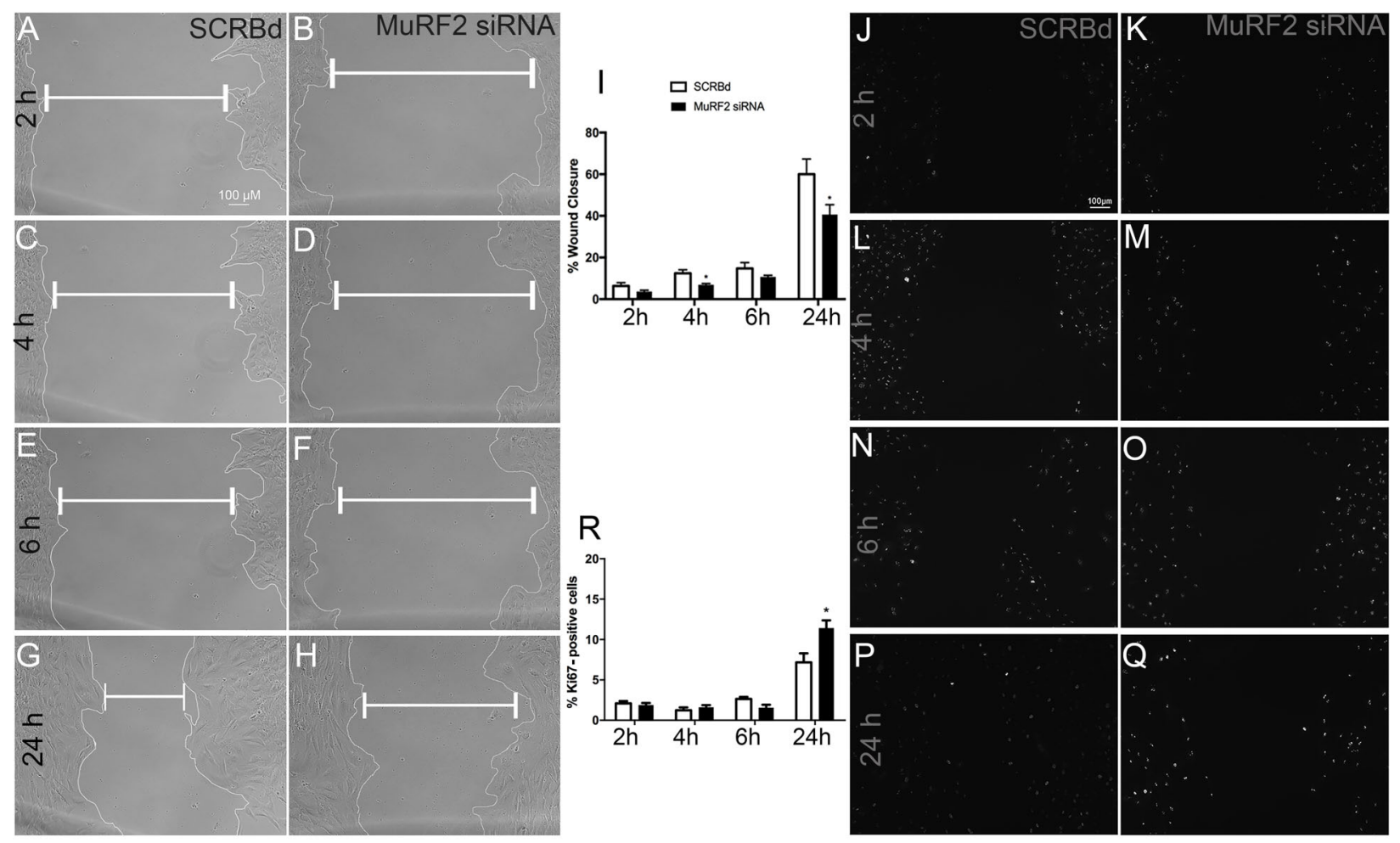

Figure 3. Knock-down of MuRF2 decreased skeletal muscle fibroblast wound healing capacity. Phase contrast microscope image from skeletal muscle fibroblasts in wound healing assay at different time points $(2,4,6$, and $24 \mathrm{~h})$. Skeletal muscle fibroblasts treated with scrambled (SCRBd) (A, C, E, and G) and with MuRF2 siRNA (B, D, F, and H); I: Percentage of wound closure obtained from the phase contrast microscope. Ki67-positive nuclei in skeletal muscle fibroblasts submitted to wound healing assay at 2, 4, 6 and $24 \mathrm{~h} \mathrm{(J-Q).}$ Skeletal muscle fibroblasts treated with SCRBd (J, L, N, and P) and with MuRF2 siRNA (K, M, O, and Q). R: Percentage of Ki67-positive cells. Data are reported as means \pm SE. ${ }^{*} \mathrm{P}<0.05$ vs SCRBd (t-test). Scale bar, $100 \mu \mathrm{M}$.

fibroblasts using specific antibodies. Even though we did not find a difference in Akt phosphorylation level at the Ser473 residue, we observed a strong reduction in Akt phosphorylation level at the Thr308 residue after MuRF2 siRNA compared to the scrambled group (Figure 4F, $\mathrm{H}$, and J).

Since collagen 1 is abundantly expressed and plays a key role in skeletal muscle tissue (28), we analyzed whether MuRF2 siRNA could modulate collagen mRNA levels. Our results showed that decreased MuRF2 expression in skeletal muscle fibroblasts did not cause alterations in collagen mRNA levels (Figure 4K).

\section{Discussion}

In the present study, we showed that MuRF2 plays an important role in skeletal muscle fibroblast function, notably acting on migration capacity. We have used a cell culture model of primary skeletal muscle fibroblasts and initially it was essential to certify that these cultures were devoid of myoblasts. We therefore labeled these cultures with MyoD (a myoblast marker) (29) and TCF4 (a fibroblast marker) (5). By using these markers, we assured that the cultures utilized were fibroblast enriched and contained no myoblasts. In addition, when a myogenic stimulus ( $2 \%$ horse serum) was applied to these cultures, we observed no myotube formation, further reassuring the absence of myoblasts. Finally, the cells used in the present study exhibited a flattened and stellate format, typically recognized as morphological characteristics of fibroblasts (30).

MuRF2 has been recognized as an important player in a variety of processes, such as in the ubiquitin proteasome system and autophagy, which seems to depend mainly upon poly-ubiquitination (31). Also, MuRF2 has been shown to be involved in the stabilization of skeletal muscle fiber structural proteins. For example, Pizon et al. reported that MuRF2 interacts with microtubules and this complex subsequently associates with sarcomeric myo$\sin$. Their results indicate that MuRF2 is necessary for proper sarcomere assembling (32). Another work established that MuRF2 is involved in sarcomeric stabilization also via intermediate filaments. Antisense oligonucleotide MuRF2 knock-down perturbed the stability of desmin 

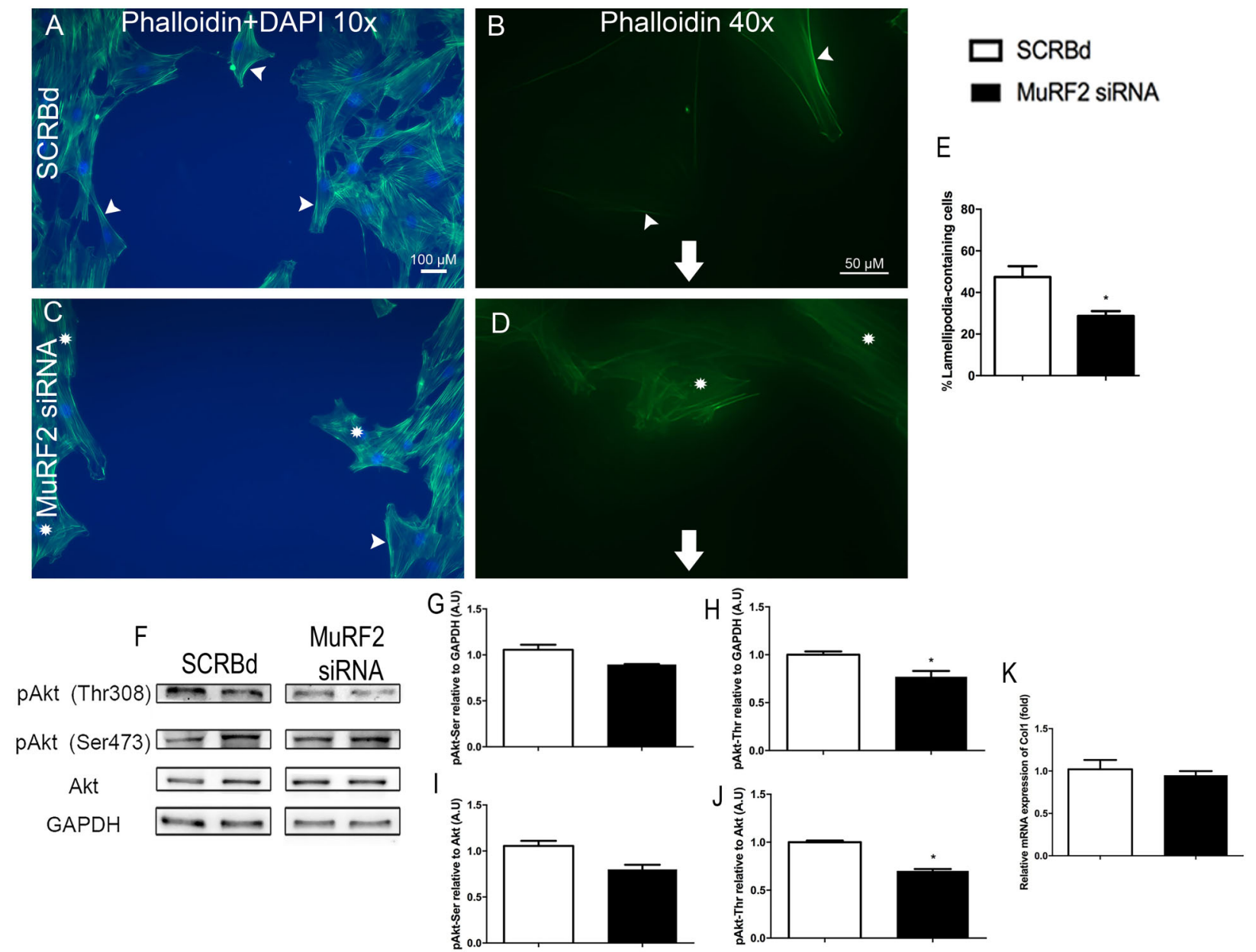

Figure 4. Knock-down of MuRF2 in skeletal muscle fibroblasts decreased lamellipodia formation. Staining for phalloidin and DAPI $24 \mathrm{~h}$ after the onset of wound healing assays at low $(\mathbf{A}$ and $\mathbf{C}, 100 \times$; bar, $100 \mu \mathrm{M})$ and high $(\mathbf{B}$ and $\mathbf{D}, 400 \times$; bar, $50 \mu \mathrm{M})$ magnifications; Skeletal muscle fibroblasts treated with scrambled (SCRBd) (A and B) and with MuRF2 siRNA (C and D). Arrowheads indicate actin stress fibers, asterisks indicate misfolded actin cytoskeleton, and arrows indicate cell migration direction. E: Quantification of lamellipodia-containing cells. F: Representative immunoblots of pAkt Ser473, pAkt Thr308, total Akt, and GAPDH in skeletal muscle fibroblasts. The images belong to the same membrane. G-J: Densitometry from western blots. K: Real-time PCR for collagen 1 (Col1) in skeletal muscle fibroblast. Data are reported means $\pm \mathrm{SE}$. ${ }^{*} \mathrm{P}<0.05$ vs $\mathrm{SCRBd}$ (t-test).

and vimentin (18). Combined, these data strongly suggest that MuRF2 works as a cytoskeleton organizer, exerting an important role in sarcomeric structure assembly and stabilization. Interestingly, a subsequent study (33) showed that MuRF2 and MuRF3 deletion in mice causes severe skeletal muscle histological alterations, including sarcomeric disarrays, reinforcing the notion that MuRF2 can be involved in cytoskeleton organization. More recently, MuRF2 was also shown to be a skeletal muscle tissue organizer in mice with MuRF1 and MuRF2 deletion (21). In spite of these data clearly establishing a connection between MuRF2 and the skeletal muscle fiber cytoskeleton, no studies had so far approached fibroblasts, which are cells extremely dependent upon their cytoskeleton to function.
MuRF2 was initially described as being expressed in skeletal and cardiac muscles along with its counterpart MuRF1 (16). Subsequent studies showed the presence of MuRF2 also in non-muscular tissues, such as a low expression of MuRF2 in the liver of pigs (34). More recently, Bian and coworkers found MuRF2 expression in several tissues, including lung, spleen, brain, liver, and kidney (35). Therefore, it is well accepted that MuRF2 is not restricted to muscle tissue, nonetheless, few studies are available addressing the localization and role of MuRF2 in specific cell types. Here, we showed that skeletal muscle fibroblasts can express MuRF2, in addition to the expected expression observed in the skeletal muscle fiber (21). Importantly, herein, MuRF2 cellular localization 
was predominantly found in the cytoplasm in skeletal muscle fibroblasts, whereas in cardiac fibroblasts, additional intense punctiform nuclear labelling was also observed. These results indicated that MuRF2 might exert differential effects depending upon which tissue fibroblasts are located. Possible mechanisms include poly-ubiquitination and subsequent degradation of specific sets of target proteins, or mono-ubiquitination, leading to changes in their activities. It would be interesting in forthcoming studies to comparatively address the impact of MuRF2 deletion in the migration capacity of fibroblasts from distinct sources.

Herein, we showed that MuRF2 silencing decreased the migratory activity of fibroblasts. In addition, we have shown that MuRF2 silencing increased cell proliferation by KI67 immunolabeling (Figure 3). We envisioned that those results combined might mean that MuRF2 could be involved in limiting cell cycle, maybe driving the cell to a more differentiated phenotype, which in turn could favor organization of cytoskeleton and consequently more migratory capacity.

In addition to skeletal muscle and cardiac fibroblasts, we also addressed mouse embryonic fibroblasts (MEFs). In MEF cells, we observed a widespread localization of MuRF2, including in the cytoplasm and the nucleus. Considering that they are embryonic fibroblasts, we interpreted that MuRF2 is an early expressed gene, participating in initial stages of cytoskeleton stability. Although currently the biological meaning of those differences in cellular localization is not clear, it could be related to key functions of those cells, such as migration and secretory activity. The control of such important functions depends upon intracellular pathways and some of them have been well described. For example, plateletderived growth factors are central chemosensory regulators of the migratory capacity in wound healing (36). In addition, $\mathrm{PI}(3,4,5) \mathrm{P}_{3}$-mediated reorganization of actin cytoskeleton can proceed by activation of Akt, which physically interacts with actin. In fact, Rahman and coworkers showed that Akt activity is crucial to the formation of actin filaments at the edge of fibroblasts during the migration process (12). Then, to address related underlying mechanisms, we investigated Akt activity, a key cytoskeleton regulator. MuRF2 siRNA caused a robust reduction in Akt phosphorylation of Thr308, strongly suggesting that MuRF2 is necessary to sustain the actin cytoskeleton functionality via maintenance of Akt activity. Our results also indicated that Akt Ser473 phosphorylation was not altered by MuRF2 silencing. Since it has been shown that Thr308 phosphorylation is accomplished by PDK1 (37), we envisioned that effects of MuRF2

\section{References}

1. Lekic PC, Pender N, McCulloch CA. Is fibroblast heterogeneity relevant to the health, diseases, and treatments of periodontal tissues? Crit Rev Oral Biol Med 1997; 8: 253268, doi: 10.1177/10454411970080030201. upon cytoskeleton remodeling depend, at least part, on PDK1 preserved activity. The precise mechanisms involved are currently not known, nonetheless it is possible that MuRF2 could poly-ubiquitinate and degrade an inhibitor of PDK1. Indeed, it is known that the protein Claudin-18 can inhibit PDK1 phosphorylation (38), therefore Claudin-18 could be considered a potential candidate for such role. Alternatively, MuRF2 could mono-ubiquitinate PDK1 keeping enzyme activity at appropriate levels. Actually, it has been shown that MuRF2 can mono-ubiquitinate PPAR $\alpha$ and PPAR $\gamma$ maintaining their activity and preserving cardiac function (39). Importantly, in the present study, we did see a correlation between decreased Akt activity and changes in cytoskeleton since MuRF2 siRNA caused about 50\% decrease in lamellipodia formation. It is largely known that lamellipodia is a major actin cytoskeleton organization process involved in cell migration $(12,27)$.

Regarding ECM production by fibroblasts, it is known that TGF- $\beta 1$ binds to its membrane receptor, which in turn activates a series of phosphorylation, promoting phosphorylation of the Smad2/3 complex. This complex also acts together with Smad4, regulating transcription of extracellular matrix proteins (40). In order to approach ECM production, we measured mRNA levels of collagen 1 and our results showed no difference in collagen $1 \mathrm{mRNA}$ in MuRF2 knocked-down skeletal muscle fibroblasts, suggesting, overall, that this E3 ligase plays an important role in fibroblast migration but not in ECM synthesis.

In conclusion, this report suggests that MuRF2 played an important role in the functional capacity of skeletal muscle fibroblasts by reducing the activation of Akt and consequently compromising the organization of the cytoskeleton. These findings provided novel insights into the regulation of skeletal muscle fibroblasts, with possible implications for conditions in which intense ECM remodeling occurs, such as during skeletal muscle regeneration, where increased migratory activity of fibroblasts is required.

\section{Acknowledgments}

We would like to thank Fundação de Amparo à Pesquisa do Estado de São Paulo (Research grant \#2015/04090-0 and Fellowship grants \#2012/13315-7 and \#2016/129412), Conselho Nacional de Desenvolvimento Científico e Tecnológico (Fellowship grant \#306915/2014-6), and the Research Executive Agency (FP7 Network Grant Sarcosi \#291834). We also thank the European Union for financial support (Horizon 2020 project \#645648, Muscle Stress Relief Network).
2. Schroeder HE, Munzel-Pedrazzoli S, Page R. Correlated morphometric and biochemical analysis of gingival tissue in early chronic gingivitis in man. Arch Oral Biol 1973; 18: 899923, doi: 10.1016/0003-9969(73)90060-5. 
3. Syverud BC, Lin E, Nagrath S, Larkin LM. Label-free, highthroughput purification of satellite cells using microfluidic inertial separation. Tissue Eng Part C Methods 2018; 24: 32-41, doi: 10.1089/ten.tec.2017.0316.

4. Chapman MA, Meza R, Lieber RL. Skeletal muscle fibroblasts in health and disease. Differentiation 2016; 92: 108-115, doi: 10.1016/j.diff.2016.05.007.

5. Murphy MM, Lawson JA, Mathew SJ, Hutcheson DA, Kardon G. Satellite cells, connective tissue fibroblasts and their interactions are crucial for muscle regeneration. Development 2011; 138: 3625-3637, doi: 10.1242/dev. 064162.

6. Tidball JG. Inflammatory processes in muscle injury and repair. Am J Physiol Regul Integr Comp Physiol 2005; 288: R345-R353, doi: 10.1152/ajpregu.00454.2004.

7. Davis ME, Gumucio JP, Sugg KB, Bedi A, Mendias CL. MMP inhibition as a potential method to augment the healing of skeletal muscle and tendon extracellular matrix. J Appl Physiol (1985) 2013; 115: 884-891, doi: 10.1152/jappl physiol.00137.2013.

8. Garg K, Corona BT, Walters TJ. Therapeutic strategies for preventing skeletal muscle fibrosis after injury. Front Pharmacol 2015; 6: 87, doi: 10.3389/fphar.2015.00087.

9. Lieber RL, Ward SR. Cellular mechanisms of tissue fibrosis. 4. Structural and functional consequences of skeletal muscle fibrosis. Am J Physiol Cell Physiol 2013; 305: C241-C252, doi: 10.1152/ajpcell.00173.2013.

10. Thievessen I, Fakhri N, Steinwachs J, Kraus V, Mclsaac RS, Gao L, et al. Vinculin is required for cell polarization, migration, and extracellular matrix remodeling in 3D collagen. FASEB J 2015; 29: 4555-4567, doi: 10.1096/ fj. 14-268235.

11. Tschumperlin DJ. Fibroblasts and the ground they walk on. Physiology (Bethesda) 2013; 28: 380-390, doi: 10.1152/ physiol.00024.2013.

12. Rahman P, Huysmans RD, Wiradjaja F, Gurung R, Ooms LM, Sheffield DA, et al. Silencer of death domains (SODD) inhibits skeletal muscle and kidney enriched inositol 5-phosphatase (SKIP) and regulates phosphoinositide 3-kinase (PI3K)/Akt signaling to the actin cytoskeleton. $J$ Biol Chem 2011; 286: 29758-29770, doi: 10.1074/jbc. M111.263103.

13. Deng S, Huang C. E3 ubiquitin ligases in regulating stress fiber, lamellipodium, and focal adhesion dynamics. Cell Adh Migr 2014; 8: 49-54, doi: 10.4161/cam.27480.

14. Hershko A, Ciechanover A. The ubiquitin system. Annu Rev Biochem 1998; 67: 425-479, doi: 10.1146/annurev.biochem. 67.1.425.

15. Hershko A. The ubiquitin system for protein degradation and some of its roles in the control of the cell-division cycle (Nobel lecture). Angew Chem Int Ed Engl 2005; 44: 59325943, doi: 10.1002/anie.200501724.

16. Spencer JA, Eliazer S, Ilaria RL Jr, Richardson JA, Olson EN. Regulation of microtubule dynamics and myogenic differentiation by MURF, a striated muscle RING-finger protein. J Cell Biol 2000; 150: 771-784, doi: 10.1083/jcb. 150.4.771

17. Centner T, Yano J, Kimura E, McElhinny AS, Pelin K, Witt $\mathrm{CC}$, et al. Identification of muscle specific ring finger proteins as potential regulators of the titin kinase domain. $J \mathrm{Mol} B i \mathrm{l}$ 2001; 306: 717-726, doi: 10.1006/jmbi.2001.4448.
18. McElhinny AS, Perry CN, Witt CC, Labeit S, Gregorio CC. Muscle-specific RING finger-2 (MURF-2) is important for microtubule, intermediate filament and sarcomeric M-line maintenance in striated muscle development. J Cell Sci 2004; 117: 3175-3188, doi: 10.1242/jcs.01158.

19. Rando TA, Blau HM. Primary mouse myoblast purification, characterization, and transplantation for cell-mediated gene therapy. J Cell Biol 1994; 125: 1275-1287, doi: 10.1083/ jcb.125.6.1275.

20. Baptista IL, Silvestre JG, Silva WJ, Labeit S, Moriscot AS. FoxO3a suppression and VPS34 activity are essential to anti-atrophic effects of leucine in skeletal muscle. Cell Tissue Res 2017; 369: 381-394, doi: 10.1007/s00441-0172614-z.

21. Moriscot AS, Baptista IL, Silva WS, Silvestre JG, Adams V, Gasch A, et al. MuRF1 and MuRF2 are key players in skeletal muscle regeneration involving myogenic deficit and deregulation of the chromatin-remodeling complex. JCSM Rapid Communications 2019; 2: 25, doi: 10.1002/j.26171619.2019.tb00010.x.

22. Barreto-Chaves ML, Anéas I, Krieger JE. Glucocorticoid regulation of angiotensin-converting enzyme in primary culture of adult cardiac fibroblasts. Am J Physiol Regul Integr Comp Physiol 2001; 280: R25-R32, doi: 10.1152/ ajpregu.2001.280.1.R25.

23. Munhoz AC, Riva P, Simoes D, Curi R, Carpinelli AR. Control of insulin secretion by production of reactive oxygen species: study performed in pancreatic islets from fed and 48-hour fasted wistar rats. PLoS One 2016; 11: e0158166, doi: 10.1371/journal.pone.0158166.

24. Fuentes-Calvo I, Crespo P, Santos E, Lopez-Novoa JM, Martinez-Salgado C. The small GTPase N-Ras regulates extracellular matrix synthesis, proliferation and migration in fibroblasts. Biochim Biophys Acta 2013; 1833: 2734-2744, doi: 10.1016/j.bbamcr.2013.07.008.

25. Mackey AL, Magnan M, Chazaud B, Kjaer M. Human skeletal muscle fibroblasts stimulate in vitro myogenesis and in vivo muscle regeneration. J Physiol 2017; 595: 51155127, doi: 10.1113/JP273997.

26. Perera S, Holt MR, Mankoo BS, Gautel M. Developmental regulation of MURF ubiquitin ligases and autophagy proteins nbr1, p62/SQSTM1 and LC3 during cardiac myofibril assembly and turnover. Dev Biol 2011; 351: 46-61, doi: 10.1016/j.ydbio.2010.12.024

27. Veland IR, Montjean R, Eley L, Pedersen LB, Schwab A, Goodship J, et al. Inversin/Nephrocystin-2 is required for fibroblast polarity and directional cell migration. PLoS One 2013; 8: e60193, doi: 10.1371/journal.pone.0060193.

28. Light $\mathrm{N}$, Champion AE. Characterization of muscle epimysium, perimysium and endomysium collagens. Biochem $J$ 1984; 219: 1017-1026, doi: 10.1042/bj2191017.

29. Tapscott SJ. The circuitry of a master switch: Myod and the regulation of skeletal muscle gene transcription. Development 2005; 132: 2685-2695, doi: 10.1242/dev.01874.

30. Ravikanth M, Soujanya P, Manjunath K, Saraswathi TR, Ramachandran CR. Heterogenecity of fibroblasts. J Oral Maxillofac Pathol 2011; 15: 247-250, doi: 10.4103/0973$029 \times .84516$.

31. Pizon V, Rybina S, Gerbal F, Delort F, Vicart P, Baldacci G, et al. MURF2B, a novel LC3-binding protein, participates with MURF2A in the switch between autophagy and ubiquitin 
proteasome system during differentiation of $\mathrm{C} 2 \mathrm{C} 12$ muscle cells. PLoS One 2013; 8: e76140, doi: 10.1371/journal. pone.0076140.

32. Pizon V, lakovenko A, Van Der Ven PF, Kelly R, Fatu C, DO Furst, et al. Transient association of titin and myosin with microtubules in nascent myofibrils directed by the MURF2 RING-finger protein. J Cell Sci 2002; 115: 4469-4482, doi: 10.1242/jcs.00131.

33. Lodka D, Pahuja A, Geers-Knorr C, Scheibe RJ, Nowak M, Hamati $J$, et al. Muscle RING-finger 2 and 3 maintain striated-muscle structure and function. J Cachexia Sarcopenia Muscle 2016; 7: 165-180, doi: 10.1002/jcsm.12057.

34. Shen H, Zhao SH, Cao JH, Li XY, Fan B. Porcine MuRF2 and MuRF3: molecular cloning, expression and association analysis with muscle production traits. Mol Biol Rep 2011; 38: 5115-5123, doi: 10.1007/s11033-010-0659-0.

35. Bian H, Gao S, Zhang D, Zhao Q, Li F, Li X, et al. The E3 ubiquitin ligase MuRF2 attenuates LPS-induced macrophage activation by inhibiting production of inflammatory cytokines and migration. FEBS Open Bio 2018; 8: 234-243, doi: 10.1002/2211-5463.12367.
36. Andrae J, Gallini R, Betsholtz C. Role of platelet-derived growth factors in physiology and medicine. Genes Dev 2008; 22: 1276-1312, doi: 10.1101/gad.1653708.

37. Sarbassov DD, Guertin DA, Ali SM, Sabatini DM. Phosphorylation and regulation of Akt/PKB by the rictor-mTOR complex. Science 2005; 307: 1098-1101, doi: 10.1126/ science. 1106148 .

38. Shimobaba S, Taga S, Akizuki R, Hichino A, Endo S, Matsunaga $T$, et al. Claudin-18 inhibits cell proliferation and motility mediated by inhibition of phosphorylation of PDK1 and Akt in human lung adenocarcinoma A549 cells. Biochim Biophys Acta 2016; 1863: 1170-1178, doi: 10.1016/ j.bbamcr.2016.02.015.

39. He J, Quintana MT, Sullivan J, Parry TL, Grevengoed TJ, Schisler JC, et al. MuRF2 regulates PPAR $\gamma 1$ activity to protect against diabetic cardiomyopathy and enhance weight gain induced by a high fat diet. Cardiovasc Diabetol 2015; 14: 97, doi: 10.1186/s12933-015-0252-x.

40. Biernacka A, Dobaczewski M, Frangogiannis NG. TGF-beta signaling in fibrosis. Growth Factors 2011; 29: 196-202, doi: 10.3109/08977194.2011.595714. 